\title{
Icacinaceae s.l. da Mata Atlântica do Nordeste, Brasil
}

\author{
Icacinaceae s.I. of Atlantic forest from Northeastern of Brazil
}

\author{
Bruno S. Amorim ${ }^{1,4}$, Anderson Alves-Araújo ${ }^{1,2}$, Rodrigo Duno de Stefano ${ }^{3}$ \& Marccus Alves ${ }^{1}$
}

\begin{abstract}
Resumo
Icacinaceae $s$. $l$. na circunscrição atual é reconhecida como quatro famílias distintas: Cardiopteridaceae, Icacinaceae S.S., Stemonuraceae e Pennantiaceae. Cardiopteridaceae, Icacinaceae s.s. e Stemonuraceae têm representantes nas florestas tropicais da América do Sul. Na parte nordeste do Domínio da Floresta Atlântica estas famílias são representadas pelas seguintes espécies: Citronella paniculata (Mart.) R.A. Roward, Dendrobangia boliviana Rusby, Discophora guianensis Miers, Emmotum affine Miers, Leretia cordata Vell. e Pleurisanthes simpliciflora Sleumer. Tais espécies apresentam distribuição disjunta entre as Florestas Atlântica e Amazônica, exceto por Emmotum affine que é endêmica da Floresta Atlântica. Chave de identificação, ilustrações, comentários sobre a distribuição geográfica, habitats e caracteres diagnósticos das espécies são apresentados.

Palavras-chave: Cardiopteridaceae, Neotrópicos, Stemonuraceae, taxonomia.
\end{abstract}

\begin{abstract}
Icacinaceae s.l. according to currently circumscription is treated as four distinct families: Cardiopteridaceae, Icacinaceae s.s., Stemonuraceae and Pennantiaceae. Cardiopteridaceae, Icacinaceae s.s. and Stemonuraceae are represented in tropical forests of South America. Along the northeastern part of the Atlantic Forest domain these families are represented by the following species: Citronella paniculata (Mart.) R.A. Roward, Dendrobangia boliviana Rusby, , Discophora guianensis Miers, Emmotum affine Miers, Leretia cordata Vell., and Pleurisanthes simpliciflora Sleumer. The species show a disjunct distribution between the Atlantic and Amazon Forests, except for Emmotum affine which is endemic to Atlantic Forest. Identification key, illustrations as well as commentaries about geographic distribution, habitats and diagnostic characteristics are presented.
\end{abstract}

Key words: Cardiopteridaceae, Neotropics, Stemonuraceae, taxonomy.

\section{Introdução}

Icacinaceae sensu lato é uma família amplamente distribuída nas regiões tropicais e subtropicais do mundo (Duno-de-Stefano 2004), com 34 gêneros e cerca de 300 espécies (Stevens 2001). Seus representantes podem ser encontrados nos mais diversos habitats, porém com pouca relevância na estrutura da comunidade arbórea (Duno-de-Stefano 2004). Os gêneros Emmotum Desv. ex Ham. (13 spp.), Citronella D. Don (12 spp.), Pleurisanthes Baill. (7 spp.), Mappia Jacq. (6 spp.) e Calatola Standl., (5 spp.) destacam-se por apresentarem maior riqueza de espécies dentre os representantes neotropicais (Duno-de-Stefano
2004; Duno-de-Stefano \& Fernández-Concha 2011; Govaerts 2011).

A taxonomia da família, principalmente das espécies da América tropical, é considerada complexa devido à lacuna do conhecimento morfológico das mesmas (Duno-de-Stefano 2004). Segundo Karehed (2001) e APG III (2009), os gêneros anteriormente considerados como relacionados em Icacinaceae s.l., agora estão melhor posicionados e distribuídos em quatro famílias distintas, a saber: Icacinaceae sensu stricto (Asterídeas - posição incerta), Cardiopteridaceae e Stemonuraceae (Asterídeas II - Aquifoliales) e Pennantiaceae (Asterídeas II - Apiales).

\footnotetext{
${ }^{1}$ Universidade Federal de Pernambuco, Depto. Botânica, Lab. Morfo-Taxonomia Vegetal - MTV, Av. Prof. Moraes Rego 1235, Cidade Universitária, 50670-901, Recife, PE, Brasil.

${ }^{2}$ Universidade Federal do Espírito Santo, Depto. Ciências Agrárias e Biológicas, Rod. BR 101 Norte, km 60, Bairro Litorâneo, 29932-540, São Mateus, ES, Brasil

${ }^{3}$ Centro de Investigación Científica de Yucatán, A.C. Calle 43 No. 130, Colonia Chuburná de Hidalgo, CVP 97200, Mérida, Yucatán, México.

${ }^{3}$ Autor para correspondência: brunosarim@yahoo.com.br.
} 
Icacinaceae s.l. é uma família de difícil distinção vegetativa por pertencer a um complexo grupo de plantas que apresentam folhas inteiras e simples, alternas e as estípulas ausentes; as flores são pequenas e simpétalas, e geralmente possuem frutos drupáceos, às vezes samaróides e unisseminados (Souza \& Lorenzi 2008; Stevens et al. 2001). Porém na Floresta Atlântica nordestina a família é representada por espécies em gêneros distintos, o que torna possível a distinção de suas espécies através apenas de seus caracteres vegetativos.

Icacinaceae sensu stricto é pantropical e possui 20 gêneros e 150 espécies. No Brasil ocorrem seis gêneros e 23 espécies distribuídas majoritariamente na Floresta Amazônica, mas podem também ser encontradas na Floresta Atlântica e nos Cerrados (Duno-de-Stefano \& Amorim 2011b).

Cardiopteridaceae e Stemonuraceae são famílias pantropicais, ocorrentes em áreas de florestas úmidas e morfologicamente muito semelhantes às Icacinaceae. Cardiopteridaceae possui cinco gêneros e aproximadamente 50 espécies, das quais, nove podem ser encontradas no Brasil. Stemonuraceae é constituída por 12 gêneros e cerca de 80 espécies, sendo apenas uma é registrada para o Brasil (Discophora guianensis Miers) (Souza \& Lorenzi 2008; Duno-de-Stefano \& Amorim 2011a; Walter 2011).

Apesar da maior diversidade taxonômica de Icacinaceae s.l. ser indicada para a floresta Amazônica (Barroso et al. 1991), para o domínio da Floresta Atlântica brasileira são citadas entre cinco e 15 espécies, ou seja, mais de $60 \%$ da diversidade encontrada na região Amazônica, pertencentes às três famílias (Groppo 2009; Duno-de-Stefano \& Amorim 2011a, b; Walter 2011).

Estudos taxonômicos em escala neotropical foram desenvolvidos com floras e revisões para os grupos de Icacinaceae s.l. (de Roon 1994; Dunode-Stefano 2007; Howard 1992, 1942; Howard \& Duno-de-Stefano 1999; Woodson et al. 1976,). Tratando dos representantes brasileiros, estudos foram iniciados com a Flora brasiliensis (Engler 1872) e posteriormente ampliados por Mazine et al. (2005) para o estado de São Paulo.

Para a Região Nordeste do Brasil estudos taxonômicos envolvendo as famílias são inexistentes, porém os trabalhos de Barbosa et al. (2006) e Ferraz \& Rodal (2006) listam espécies pertencentes às três famílias e confirmam a frequente presença das mesmas em listas florísticas para a região.
Durante estudos para a flora do Nordeste foram constatadas lacunas nas coleções de Cardiopteridaceae, Icacinaceae s.s. e Stemonuraceae em diversos herbários regionais, particularmente no que se refere à correta identificação taxonômica das amostras. Seguindo o direcionamento proposto para o estudo botânico de famílias para a Floresta Atlântica na região Nordeste do Brasil, a exemplo de Quiinaceae (Alves-Araújo et al. 2010), este trabalho visa propiciar um melhor conhecimento das famílias na Região, além de subsidiar a reedição da Lista de Espécies da Flora do Brasil.

\section{Material e Métodos}

As informações foram obtidas através das amostras coletadas em diversos Estados do Nordeste e analisadas com auxílio de estéreo-micoscópio e as informações complementares provenientes das etiquetas das exsicatas dos principais acervos botânicos regionais, nacionais e internacionais (ASE, BHCB, BM, CEPEC, DIAM, EAC, F, G, HST, HUH, IPA, JPB, K, MAC, MO, NY, P, PEUFR, RB, TEPB, UESB, UFP, UFRN, US e VEM; siglas de acordo com Thiers 2011). Foi utilizada bibliografia específica para identificação e consulta às imagens do material-tipo disponibilizado online JSTOR ( $<$ http://plants.jstor.org/ $>$ ). As amostras coletadas estão depositadas no herbário UFP. São apresentados apenas os sinônimos de maior relevância para as espécies. Os termos botânicos seguiram o proposto por Harris \& Harris (2001).

\section{Resultados e Discussão}

Foram registradas duas espécies de Cardiopteridaceae (Citronella paniculata (Mart.) R.A.Howard e Dendrobangia boliviana Rusby)), três de Icacinaceae s.s. (Emmotum affine Miers, Leretia cordata Vell. e Pleurisanthes simpliciflora Sleumer) e uma de Stemonuraceae (Discophora guianensis Miers). Dentre elas, apenas Emmotum affine é endêmica da Floresta Atlântica, na qual as demais espécies apresentam distribuição disjunta entre as Florestas Atlântica e Amazônica.

A Região Nordeste detém $40 \%$ das espécies (6/15 spp.) listadas para o domínio da Mata Atlântica no Brasil (Duno-de-Stefano \& Amorim 2011a; Duno-de-Stefano \& Amorim 2011b; Walter 2011), sendo a Bahia o estado mais diverso com seis táxons, seguido por Pernambuco (3 spp.), Alagoas e Paraíba (2 spp. cada) e Sergipe (1 spp.). 
De acordo com a lista de espécies ameaçadas da IUCN (IUCN 2011), nenhum dos táxons aqui analisados encontra-se sob algum grau de ameaça. No entanto, dada a raridade das amostras nas coleções botânicas e à constante perda de habitats da Mata Atlântica no Brasil, estudos populacionais tornam-se extremamente necessários para indicação dos níveis de ameaça mais próximos da realidade.

\section{Chave para identificação de Icacinaceae s.l. do Domínio da Floresta Atlântica no Nordeste do Brasil.}

1. Ramos e folhas com indumento lepidoto-estrelado; flores com corola unida em um tubo curto com estames adnados a este 2. Dendrobangia boliviana (Cardiopteridaceae)

1'. Ramos e folhas glabros ou com indumento, nunca lepidoto-estrelado; flores nunca como a anterior.

2. Lianas.

3. Ramos com lenticelas; tricomas em forma de " $\mathrm{t}$ " nos ramos jovens, folhas, inflorescência e flores; fruto indumentado internamente 5. Leretia cordata (Icacinaceae s.s.)

3'. Ramos sem lenticelas; tricomas simples nos ramos jovens, folhas e inflorescência e flores; fruto não indumentado internamente.

4. Folhas $>15 \mathrm{~cm}$ compr., frutos costados ...... 3. Discophora guianensis (Stemonuraceae)

4'. Folhas $\leq 15 \mathrm{~cm}$ compr., frutos não costados

6. Pleurisanthes simpliciflora (Icacinaceae s.s.)

2'. Árvores ou arbustos com ramos decumbentes.

5. Domácias na face abaxial das folhas ............. 1. Citronella paniculata (Cardiopteridaceae)

5'. Domáceas ausentes na face abaxial das folhas.

6. Folhas $\leq 15 \mathrm{~cm}$ compr., com indumento dourado na face abaxial; pétalas com indumento laranja na face interna (material herborizado); fruto subgloboide, não costado

4. Emmotum affine (Icacinaceae s.s.)

6'. Folhas $>15 \mathrm{~cm}$ compr., glabras ou com indumento hialino na face abaxial; pétalas glabras ou com indumento hialino na face interna; fruto elipsoide, costado

3. Discophora guianensis (Stemonuraceae)

1. Citronella paniculata (Mart.) R.A.Howard, J. Arnold Arbor. 21(4): 473. 1940. Villaresia paniculata (Mart.) Miers, Ann. Mag. Nat. Hist., ser. 3 9: 116. 1862. Citronella megaphylla (Miers) R.A.Howard, J. Arnold Arbor. 21(4): 472. 1940.

Fig. 1a-b

Árvores até $8 \mathrm{~m}$ alt. ocorrentes na América do Sul e com distribuição disjunta entre as Florestas Amazônica (Venezuela, Brasil) e Atlântica (Brasil, Paraguai e Argentina). No domínio da Mata Atlântica do Brasil, a espécie possui ampla distribuição e para o Nordeste é registrada para florestas estacionais e ombrófilas densas na Bahia (Duno-de-Stefano 2006; Duno-de-Stefano \& Amorim 2011a).

Diferencia-se das demais espécies por apresentar ramos glabros, lenticelas ausentes; folhas $8-15(23) \times 4-8 \mathrm{~cm}$ com nervura principal sulcada, secundária de 6-8 pares, presença de domáceas nas folhas totalmente expandidas e localizadas nos vértices das venações primária e secundária da face foliar abaxial; pecíolo 0,5-2 cm compr., sulcado, glabro; frutos $1,7 \times 1,1 \mathrm{~cm}$, elipsoides, não costados, glabros.
Popularmente conhecida como carne-de-vaca ou perobossu (Duno-de-Stefano \& Amorim 2011a). Material examinado: BAHIA: Ilhéus, área do CEPEC, 11.VIII.1981, fl., T.S. Santos 3629 et al. (CEPEC); 16.IX.1981, fr., T.S. Santos 3670 et al. (CEPEC). Material adicional: BRASIL. PARANÁ: São Gerônimo da Serra, São João Nogueira, 11.IX.1999, fr., C. Medri 850 et al. (HST). RIO DE JANEIRO: Paraty, 29.VI.1993, fl., $R$. Marquete 1083 et al. (CEPEC, RB).

2. Dendrobangia boliviana Rusby, Mem. Torrey Bot. Club 6(1): 19-20. $1896 . \quad$ Fig. 1c

Árvores até $12 \mathrm{~m}$ alt., registrada para as Regiões Norte e Nordeste do Brasil (Duno-de-Stefano 2006, Duno-de-Stefano \& Amorim 2011a). A espécie ocorre desde a Costa Rica e Panamá até a Bolívia e Brasil, sendo disjunta entre a região Amazônica e a Floresta Atlântica nordestina, onde habita florestas ombrófilas densas na Bahia (Duno-de-Stefano \& Amorim 2011a).

Diferencia-se das demais espécies principalmente pelos ramos e folhas com indumento lepidoto-estrelado; folhas $8-18 \times 4,5-7,8 \mathrm{~cm}$, nervura principal plano-convexa, secundária $8-10$ 

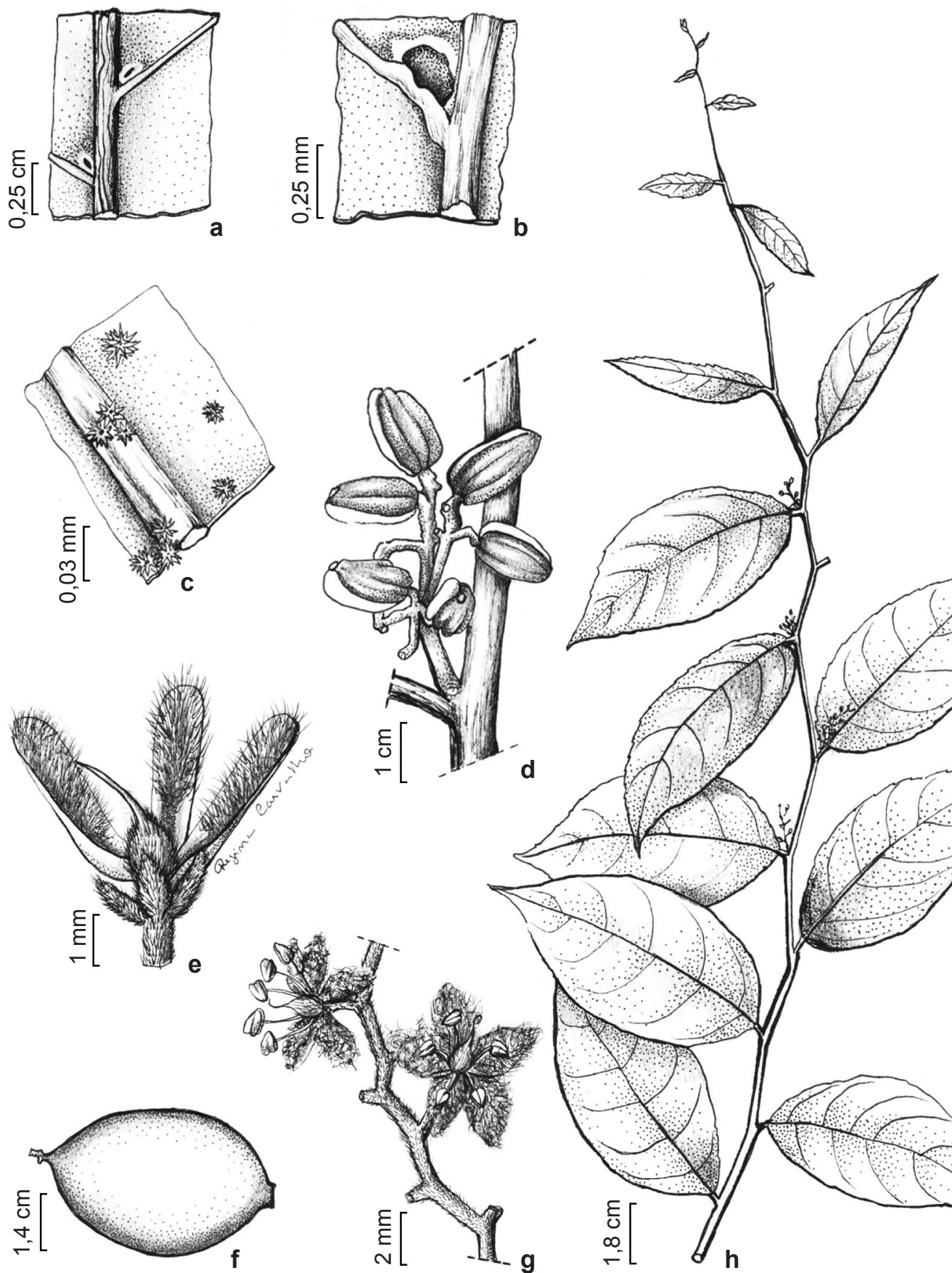
pares; pecíolo 0,8-1,2 cm, plano-convexo; flores com corola unida em um tubo curto com estames adnados a este, e fruto $2-1,5 \times 10-1,5 \mathrm{~cm}$ elipsoide, glabro.

Popularmente conhecida como pau-de-cubiú (Duno-de-Stefano \& Amorim 2011a).

Material selecionado: BAHIA: Teixeira de Freitas, estrada para Caravelas, 10.VIII.1972, fl., T.S. Santos 2347 et al (A). Material adicional: BRASIL. PARÁ: Bosque Rodrigues Alves, 24.III.1982, fl., A.L. Mesquita 446 et al (HST). Belém, Água Preta, 07.V.1946, fr., A. Ducke 1944 (INPA, NY, US).

3. Discophora guianensis Miers, Ann. Mag. Nat. Hist., ser. 2, 10: 119. 1852. Discophora froesii Pires, Bol. Tecn. Inst. Agron. N. 38: 28. 1960.

Fig. 1d

Arbustos a árvores 3-10 m alt. ou lianas com registros para as Regiões Norte, Nordeste e CentroOeste do Brasil (Duno-de-Stefano 2006, Walter 2011). Ocorre da Costa Rica a Bolívia e Brasil, sendo disjunta entre as florestas Amazônica e Atlântica do Nordeste do Brasil (AL, BA e PE) (Walter 2011). No domínio da Mata Atlântica, D. guianensis pode ser encontrada em florestas estacionais semideciduais e florestas ombrófilas densas submontanas.

Diferencia-se por apresentar ramos pubescentes, sem lenticelas; folhas 20-27,5 × 6,5-9 cm, pubescentes, nervura principal sulcada, secundária 7-9 pares; pecíolo sulcado adaxialmente e pubescentes; frutos $1,3-1,5 \times 0,7-0,9 \mathrm{~cm}$, elipsoides, 8-10-costados.

Popularmente conhecida como pombinho (Walter 2011).

Material selecionado: ALAGOAS: Ibateguara, Coimbra, 12.III.2003, fl., M. Oliveira 1306 et al. (MAC). BAHIA: Itagí, Fazenda Palestina, 24.VIII.2011, fr., B.S. Amorim 1018 et al. (UFP). Porto Seguro, Reserva da Brasil Holanda de Indústrias S/A, 07.IV.1984, fl., J.G. Jardim 384 et al. (CEPEC). PERNAMBUCO: Igarassu, Usina São José, 06.V.2009, fl., A. Alves-Araújo 1238 et al. (ASE, UFP);

4. Emmotum affine Miers, Ann. Mag. Nat. Hist.,ser. 2, 10: 180. 1852.

Fig. 1e

Arbustos a árvores até $10 \mathrm{~m}$ alt., com registro para as Regiões Nordeste e Sudeste do Brasil, da Paraíba ao Espírito Santo (Duno-de-Stefano 2006; Duno-de-Stefano \& Amorim 2011b; Duno-deStefano \& Fernández-Concha 2011). Dentre as espécies estudadas, é a única endêmica da Floresta Atlântica. Comum nas Florestas ombrófilas densas do sul da Bahia, além da ocorrência em restingas de Alagoas, Paraíba, Sergipe e Pernambuco (Dunode-Stefano \& Amorim 2011b).
Diferencia-se pelos ramos pubescentes, com tricomas hialinos, sem lenticelas; folhas 3,5-13 $\times$ 2-8 cm, com tricomas dourados na face abaxial, margem revoluta, nervura principal sulcada, as secundária 6-8 pares; pecíolo sulcado na face adaxial; pétalas pubescentes, tricomas hialinos na face externa, lanulosos-alaranjados (quando herborizados) na face interna; frutos $1,3 \times 1 \mathrm{~cm}$, subgloboides, não costados, pubescentes.

Popularmente conhecida como aderno, aderno da praia, guagirú e louro da caatinga (Dunode-Stefano \& Amorim 2011b).

Material selecionado: ALAGOAS: Marechal Deodoro, APA de Santa Rita, 3.IV.2002, fl. e fr., R.P Lyra-Lemos 6503 et al. (MAC). Piaçabuçu, Soares, 18.XI.1987, fl., G.L. Esteves 1969 et al. (MAC). BAHIA: Belmonte, Estação Experimental Gregório Bondai, 2. IV. 2003, fr., P. Fiaschi 1452 et al. (CEPEC). Cairu, Ilha da Boipeba, 17.VIII.2008, fl., J.G. Jardim 5347 et al. (CEPEC). PARAÍBA: Conde, Jacumã, 15.IX.2005, fl., fr., E.A. Rocha 1366 et al. (CEPEC). PERNAMBUCO: Goiana, Engenho Itapirema da Mata, 18.IV.1967, fr., A. Lima 674991 et al. (IPA). Paulista, Praia da Coneição, 16.XI.1961, fl., S. Tavares 862 et al. (HST). SERGIPE: Pirambu, 3.IV.1984, fl., G. Viana 914 et al. (ASE); Santa Luzia do Itanhi, 9.X.1993, fl. S.C. Sant'Ana 422 et al. (ASE).

5. Leretia cordata Vell., Fl. Flumin. 99, t. 2a, 3. 1825[1829]. Mappia cordata (Vell.) Engl., Fl. bras. 12(2): 50. 1872.

Fig. $1 \mathrm{f}-\mathrm{g}$.

Liana a arbusto escandente, 8,5-15 m alt., com registro para as Regiões Norte, Nordeste e Sudeste do Brasil (Duno-de-Stefano 2006, 2010). Amplamente distribuída desde a Costa Rica e Panamá ao Brasil. Disjunta entre as florestas Amazônica e Atlântica (BA, ES, MG e RJ) (Duno-de-Stefano \& Amorim 2011b).

Diferencia-se pelos ramos lenticelados, com tricomas em forma de " $\mathrm{t}$ " nos ramos jovens, folhas, inflorescência e flores; folhas com 4,5-16(20) $\mathrm{cm} \times 4,5-6 \mathrm{~cm}$, pétalas com tricomas lanulosos na face interna e hialinos, frutos com 4-6 × 3-4 $\mathrm{cm}$, subgloboides a elipsoides, superfície glabra, indumentado internamente.

Popularmente conhecida como puca yacua e/ ou surucuína (Duno-de-Stefano \& Amorim 2011b). Material selecionado: BAHIA: Amargosa: Serra do Timbó, 26.I.2007, fl., D. Cardoso 1609 et al. (CEPEC). Santa Maria Eterna, estrada próximo a Canavieiras, 12.V.1970, fl., T.S. Santos 780 et al. (RB);.Una, Reserva Biológica de Uma, 5.V.2005, fr., J.L. Paixão 396 et al (CEPEC).

Material adicional: ESPÍRITO SANTO: Guarapari, Rodovia do Sol, 23.II.1988, fl., J.R. Pirani 2421 et al. (NY). RIO DE JANEIRO: Paraty, APA Cairucu, 20.X.1993, fr., R. Marquete 1269 et al. (RB). 
6. Pleurisanthes simpliciflora Sleumer, Notizbl. Bot. Gart. Berlin-Dahlem 15: 256. 1940. Fig. 1h

Trepadeira, com distribuição disjunta entre as Florestas Amazônica e Atlântica (Duno-deStefano \& Amorim 2011b). No domínio da Mata Atlântica, tem distribuição para as Regiões Sudeste (MG) e Nordeste (BA, PB e PE) e ocorre em áreas de restinga e Florestas estacionais semideciduais (Duno-de-Stefano \& Amorim 2011b).

Reconhecida pelos ramos pubescentes, com tricomas hialinos, sem lenticelas; folhas com 4,8-8 $\times 2,5-5,5 \mathrm{~cm}$, face abaxial com indumento hirsuto de tricomas simples; nervura principal planoconvexa, secundárias $6-8$ pares, pecíolo $2-5 \mathrm{~mm}$ compr., plano-convexo adaxialmente; frutos $2 \times 1,2$ $\mathrm{cm}$, elipsoides, não costados, glabros.

Morfologicamente relacionada a Pleurisanthes brasiliensis (Val.) Tiegh., da qual foi constatada ocorrência nas Florestas ombrófilas da Bahia (Dunode-Stefano \& Amorim 2011b). Porém, por carência de material a ser analisado, no presente estudo não foi possível diagnosticar divergências morfológicas entre as duas espécies, em que o táxon é tratado como P. simpliciflora.

Material selecionado: BAHIA: Maraú, 05.VIII.2002, fr., W.W. Thomas 13080 et al. (CEPEC). Una, estrada Ilhéus/ Uma, 02.XII.1981, fl., A.M. Carvalho 876 et al. (CEPEC). PARAÍBA: Cupissura, 29.VII.1958, fr., A. Lima 58-3225 (IPA). PERNAMBUCO: Goiana, Engenho Maçaranduba, 14.II.1968, fl., O.C. Lira 68-187 et al. (IPA).

\section{Agradecimentos}

Os autores agradecem aos curadores dos diversos herbários visitados e/ou consultados; ao financiamento do CNPq, U. S. National Science Foundation (DEB-0946618), Velux Stiftung, e Beneficia Foundation; à Regina Carvalho, pela excelente qualidade das ilustrações botânicas e aos integrantes do laboratório de Morfo-Taxonomia Vegetal - UFPE, pelo constante auxílio.

\section{Referências}

Alves-Araújo, A; Lucena, M.F.A. \& Alves, M. 2010. Quiinaceae do Nordeste. Rodriguésia 61: 415-420.

APG III. 2009. An update of the angiosperm phylogeny group classification for the orders and families of flowering plants: APG III. Botanical Journal of the Linnean Society 161: 105-121.

Barbosa, M.R.V.; Sothers, C.; Mayo, S.; GamarraRojas, C.F.L. \& Mesquita, A.C. 2006. Checklist das plantas do nordeste brasileiro: angiospermas e gymnospermas. Ministério de Ciência e Tecnologia, Brasília. 156p.
Barroso, G.M.; Guimarães, E.F.; Ichaso, C.L.F.; Costa, C.G. \& Peixoto, A.L. 1991. Sistemática de angiospermas do Brasil. Vol. 2. $2^{\text {a }}$ ed. Imprensa Universitária/ Universidade de Viçosa, Viçosa. 377p.

De Roon, A.C. 1994. 112. Icacinaceae. In: Görts-van Rijn, A.R.A. (ed.). Flora of the Guianas, ser. A, Phanerog. 16: 82-109.

Duno-de-Stefano, R. 2004. Icacinaceae. In: Smith, N.; Mori, S.A.; Henderson, A.; Stevenson, D.Wm. \& Heald, S.V. (eds.). Flowering plants of the Neotropics. Princeton University Press, Princeton. Pp. 192-193.

Duno-de-Stefano, R. 2006. Icacinaceae. In: Barbosa, M.R.V.; Sothers, C.; Mayo, S.; Gamarra-Rojas C.F.L. \& Mesquita, A.C. (orgs.). Checklist das plantas do Nordeste brasileiro: angiospermas e gymnospermas. Ministério de Ciência e Tecnologia Brasília. Pp. 77-78.

Duno-de-Stefano, R. 2007. Tratamiento taxonómico del género Dendrobangia Rusby (Cardiopteridaceae o Icacinaceae). Candollea 62: 91-103

Duno-de-Stefano, R. \& Amorim, B. $2011 \mathrm{a}$. Cardiopteridaceae. In: Forzza, R.C. et al. (eds.). Lista de espécies da flora do Brasil. Jardim Botânico do Rio de Janeiro. Disponível em $<$ http://floradobrasil.jbrj.gov. br/2011/FB000078>. Acesso em 15 Out 2011.

Duno-de-Stefano, R. \& Amorim, B. 2011b. Icacinaceae. In: Forzza, R.C. et al. (eds.). Lista de espécies da flora do Brasil. Jardim Botânico do Rio de Janeiro. Disponível em <http://floradobrasil.jbrj.gov.br/2011/ FB000135>. Acesso em 4 Dez 2011.

Duno-de-Stefano, R. \& Fernández-Concha, G.C. 2011. Morphology-inferred phylogeny and a revision of the genus Emmotum (Icacinaceae). Annals of the Missouri Botanical Garden 98: 1-27.

Engler, A. 1872. Icacineae. In: Martius, C.F.P.; Eichler,A.W. \& Urban, I. (eds.). Flora brasiliensis. Vol. 12, pars II, Fasc. 60. Frid. Fleischer, Leipzig. Pp. 41-62.

Ferraz, E.M.N. \& Rodal, M.J.N. 2006. Caracterização fisionômica-estrutural de um remanescente de floresta Ombrófila Montana de Pernambuco, Brasil. Acta Botanica Brasilica 20: 911-926.

Govaerts, R. 2011. World Checklist of Cardiopteridaceae. Facilitated by the Royal Botanic Gardens, Kew. Disponível em $<$ http://apps.kew.org/wcsp/>. Acesso em 18 Out 2011.

Groppo, M. 2009. Icacinaceae. In: Stehmann, J.R; Forzza, R.C.; Salino, A; Sobral, M.; Costa, D.P. \& Kamino, L.H.Y. (eds.). Plantas da Floresta Atlântica. Instituto de Pesquisas do jardim Botânico do Rio de Janeiro, Rio de Janeiro. Pp. 294.

Harris, J.G. \& Harris, M.W. 2001. Plant identification terminology: An illustrated Glossary. $2^{\text {nd }}$ ed. Spring Lake Publishing, Utah. 216p.

Howard, R.A. 1942. Studies of the Icacinaceae II: Humirianthera, Leretia, Mappia and Nothapodytes, valid genera of the Icacinaceae. Journal of the Arnold Arboretum 23: 55-78. 
Howard, R.A. 1992. A revision of Casimirella, including Humirianthera (Icacinaceae). Brittonia 44: 166-172.

Howard, R.A. \& Duno-de-Stefano, R. 1999. Icacinaceae .In: Berry, P.E.; Yatskievysh, K. \& Host, B.K. (eds.). Flora of the Venezuelan Guayana. Vol 5. Missouri Botanical Garden Press, St. Louis. Pp. 646-658

IUCN 2011. IUCN Red list of threatened species. Version 2011.1. Disponível em <www.iucnredlist.org>. Acesso em 9 Set 2011.

Karehed, J. 2001. Multiple origin of the Tropical Forest tree family Icacinaceae. American Journal of Botany 88: 2259-2274.

Mazine, F.F.; Souza, V.C. \& Rodrigues, R.R. 2005. Icacinaceae. In: Wanderley, M.G.L.; Shepherd, G.J.; Melhem, T.S. \& Giulietti, A.M. (ed.). Flora fanerogâmica do estado de São Paulo. Vol. 4. Instituto de Botânica, São Paulo. Pp. 227-229.

Souza, V.C. \& Lorenzi, H. 2008. Botânica sistemática: guia ilustrado para identificação das famílias de fanerógamas nativas e exóticas no Brasil, baseado em APG II. $2^{\mathrm{a}}$ ed. Instituto Plantarum, Nova Odessa. $704 p$.

Stevens, P.F. 2001 [onwards]. Angiosperm phylogeny website. Disponível em <http://www.mobot.org/ MOBOT/research/APweb/>. Acesso em 8 Set 2011.

Thiers, B. 2011 [continuously updated]. Index Herbariorum: a global directory of public herbaria and associated staff. New York Botanical Garden's Virtual Herbarium. Disponível em $<\mathrm{http}$ ://sweetgum. nybg.org/ih/>. Acesso em 6 Out 2011.

Walter, B.M.T. 2011. Stemonuraceae. In: Forzza, R.C. et al. (eds.). Lista de espécies da flora do Brasil. Jardim Botânico do Rio de Janeiro. Disponível em $<$ http://floradobrasil.jbrj.gov.br/2011/FB000228>. Acesso em 15 Out 2011.

Woodson Jr., R.E; Schery, R.W. \& Howard, R.A. 1976. Flora of Panama. Part VI. Family 106. Icacinaceae. Annals of Missouri Botanical Garden 63: 399-417.

\section{Lista de exsicatas}

Adorno, H. 45 (4). Almeida, J. 77 (1), 107 (4). Alves-Araújo, A. 1238 (3). Amaral, C. 31 (4). Amorim, A. 415 (3), 889 (6), 939 (3), 1067 (3), 1206 (3), 1275 (3), 2006 (3), 2567 (4), 2892 (1), 3303 (4), 5109 (1), 6029 (3). Amorim, B.S. 1018 (3). Andrade, P.M. 652 (5). Araújo, G. 68 (4). Araújo, G.B. 311 (4). Bautista, H.P. 1164 (4), 1183 (4). Bayma, I.A. s/n MAC 21757. Belém, R.P. 2666 (3), 3042 (4), 3305 (4), 3329 (3), 3465 (4). Blanchet, J. 1902 (4). Callejas, R. 1722 (4). Cardoso, D. 1061 (4), 1609 (5). Carneiro, E. 132 (4). Carvalho, A.M. 382 (4), 535 (4), 624 (4), 840 (4), 876 (6), 1162 (3), 3303 (4), 4129 (6), 4439 (3), 4661 (5), 4699 (5). Carvalho, G.M. 302 (3). Chagas-Mota 1421 (4). Costa, L.V. s/n BHCB 52533 (5). Costa, S.M. 238 (4). Daneu, L. 297 (3). Duarte, A.P. 8051 (4). Ducke, A. 1931 (2), 1944 (2). Esteves, G.L. 1929 (4), 1965 (4), 1969 (4), 2067 (4), 2169 (4). Eupunino, A. 152 (4). Ferreira, M.C. 845 (4), 919 (4). Fiaschi, P. 1206 (1), 1452 (4). Fonseca, M. 1067 (4), s/n, ASE-498 (4). Fróes, R.L. 20260 (5). Guedes, M.L. 4011 (4), 6591 (4), 7401 (4). Hage, J.L. 273 (4), 1201 (1), 1348 (1), 1396 (1), 2363 (4). Harley, R.M. 17055 (4), 18156 (4), 18388 (4), 25603 (4). Hatschbach, G.M. 48754 (4), 52244 (3). Hoehne, F.C. 29752 (1). Jardim, J.G. 384 (3), 2189 (4), 2982 (3), 5347 (4). Jesus, J.A. 2129. Jost, T. 108 (4). Kallunki, J. 504 (3). Kegler, A. 495 (1). Lanna, J.P. 1381 (4). Lima, A. 24-67 (4), 50-445 (4), 55-2071 (4), 58-3225 (6), 65-4312a, 67-4991 (4). Lima, A. \& Costa, Medeiros-Costa 41 (4), 71 (4). Lira, O.C. 68-187 (6). Luna-Peixoto, A. 333 (4). Lombardi, J. 3617 (5). Lopes, M.M. 921 (3). Lyra-Lemos, R.P. 263 (4), 3831 (4), 4018 (4), 4513 (4), 6503 (4), 6504 (4),. 7359 (4), 7481 (4). Macedo, G.E.L. 423 (4). Marquete, R. 1083 (1), 1269 (5). Mattos-Silva, L.A. 482 (3), 881 (4), 897 (3), 923 (4), 1837 (6), 1838 (6), 1864 (4), 1886 (6), 2216 (6), 2396 (1), 2481 (3), 2644 (4), 2696 (6). Medri, C. 850 (1). Mendes, M.J. 60 (4). Mendonça, C.V. s/n BHCB 26009. Mesquita, A.L. 446 (2). Miranda, A.M. 4520 (4), 4637 (4), 5356 (4), 5395 (4), 5848 (4). Moreira, I.S. 44 (4). Mori, S.A. 9750 (4), 9811 (3), 10185 (4), 10577 (4), 10711 (3), 10903 (4), 11696 (3), 11679 (4), 11717 (3), 11971 (4), 12122 (4), 12717 (3), 13057 (4), 14057 (4). Oliveira, M. 1306 (3). Nogueira, C.P. s/n BHCB 69556 (5). Orlandi, R.P. 183. Paixão, J.L. 392 (3), 396 (5), 1452 (3). Perdiz, R. 629 (3). Pereira, P.A. 76 (4). Pickel, B. 1037 (4), 3125 (4). Pinheiro, R.S. 139 (1), 1410 (4), 1732 (5). Pinto, G.C.P. $315 / 83$ (4). Pirani, J.R. 2421 (5), 2750 (4), 2962 (4). Plowman, T. 12781 (4). Reitz \& Klein 3348 (1). Riedel, L. 1159 (1), s/n NY 626151 (4), s/n NY 626202 (5). Rocha, E.A. 1366 (4). Rocha, R.F. 178 (4), 263 (4), 434 (4), 541 (4). Santana, F.D. 11 (3). Sant'Ana, S.C. 127 (4), 422 (4), 897 (3). Santos, E.B. 227 (3). Santos, F.S. 118 (3), 484 (4). Santos, T.S. 780 (5), 1023 (6), 2347 (2), 2862 (3), 2941 (3), 3629 (1), 3670 (1), 4037 (3), 4207 (3), 4292 (5), 4408 (3), 4552 (4). Souza, J.P. 12220 (4). Stehmann, J.R. 3854 (4), 4747 (4). Tameirão-Neto, E. 2583 (5) Tavares, S. 862 (4). Thomas, W.W. 4139 (5), 6106 (4), 6995 (3), 7138 (3), 8792 (3), 9395 (3), 9422 (6) 9875 (4), 10420 (5), 11607 (4), 12080 (3), 13080 (6). Viana, B.F. 37 (4). Viana, G. 914 (4). Vinha, S.G. 160 (4). Violatti, L.G. 223 (4). Vellozo, E. s/n CEPEC 100627 (4). Voeks, R. 80 (4). 